Article

\title{
Development of a Method for the Measurement of Human Scent Samples Using Comprehensive Two-Dimensional Gas Chromatography with Mass Detection
}

\author{
Petra Pojmanová *®D, Nikola Ladislavová and Štěpán Urban \\ Department of Analytical Chemistry, Faculty of Chemical Engineering, University of Chemistry and Technology, \\ Prague, Technická 5, 16628 Prague, Czech Republic; ladislan@vscht.cz (N.L.); urbans@vscht.cz (Š.U.) \\ * Correspondence: petra.pojmanova@vscht.cz
}

Citation: Pojmanová, P.; Ladislavová, N.; Urban, Š. Development of a Method for the Measurement of Human Scent Samples Using Comprehensive Two-Dimensional Gas Chromatography with Mass Detection. Separations 2021, 8, 232 https://doi.org/10.3390/ separations 8120232

Academic Editors: Miguel Ángel Rodríguez-Delgado and Bárbara Socas-Rodríguez

Received: 31 October 2021 Accepted: 1 December 2021 Published: 3 December 2021

Publisher's Note: MDPI stays neutral with regard to jurisdictional claims in published maps and institutional affiliations.

Copyright: (c) 2021 by the authors. Licensee MDPI, Basel, Switzerland. This article is an open access article distributed under the terms and conditions of the Creative Commons Attribution (CC BY) license (https:// creativecommons.org/licenses/by/ $4.0 /)$.

\begin{abstract}
Every human body is a source of a unique scent, which can be used for medical or forensic purposes. Human skin scent is a complex mixture of more or less volatile compounds with different chemical and physical properties, which often differ significantly in their concentrations. The most efficient technique for separating such complex samples is comprehensive two-dimensional gas chromatography $(\mathrm{GC} \times \mathrm{GC})$. This work aimed to find the optimal arrangement of a two-dimensional chromatographic system and define a suitable chromatographic method for non-targeted analysis of human scent samples. Four different chromatographic columns (non-polar Rxi-5MS and TG-5HT, medium polar Rxi-17Sil MS and Rtx-200MS) and their different configurations were tested. The best system was the $30 \mathrm{~m}$ primary column Rtx-200MS (with the $2 \mathrm{~m}$ pre-column Rtx-200MS) and the $1 \mathrm{~m}$ secondary column TG-5HT in a reverse configuration. This system achieved the highest theoretical and conditional peak capacities, optimal resolution, and the lowest number of coelutions.
\end{abstract}

Keywords: human skin scent; comprehensive two-dimensional gas chromatography; mass spectrometry

\section{Introduction}

The human scent and its molecular profile are one of the major topics of current forensic interest. The possibility of its chemical analysis and identification of the individual based on scent composition would mean a significant breakthrough in forensic practice, providing more convincing evidence than specially trained dogs [1-5]. In addition to forensic practice, monitoring changes in the molecular profile of an individual would be of great importance for the evaluation of physical or mental conditions in biometric and medical applications [6-8].

Concerning human scent, a number of publications $[9,10]$ focus mainly on volatile organic compounds (VOCs), which are largely contained in human skin scent and which can be found in all body fluids and excrement: in breath, saliva, blood, milk, urine, feces, and skin secretions (sweat and follicular fluids). In 2014, de Lacy Costello et al. [6] published a review with several hundred VOCs of the human scent. However, some authors point to the importance of low volatile compounds [11-13]. In general, the human skin scent contains several thousand different compounds, e.g., acids, alcohols, aldehydes, amides, amines, esters, hydrocarbons, ketones, heterocyclic compounds, fatty acids, and fatty acid esters. These compounds, in addition, can vary in concentrations by several orders of magnitude [12]. The qualitative and quantitative variability of the human scent is influenced by many circumstances, e.g., by the sampling condition and sampled body parts, age, sex, heredity, diet, drugs, mood and emotions, cosmetics, and environmental factors [14,15].

For the analysis of volatile compounds in real complex samples, such as human scent, conventional gas chromatography is often an insufficient separation tool. Some analytes cannot be separated during a single analysis due to their similar properties, and 
either overlapping or insufficiently distributed peaks appear in the chromatogram, causing problems for both identification and quantification.

The aim of the experiments described in this work is to find the optimal arrangement of the 2D chromatographic system and a suitable chromatographic method to achieve the best separation condition for non-targeted analysis of human scent samples.

\section{Materials and Methods}

\subsection{Materials}

Hexane (quality for GC-MS) and all standards used (see Table S1) were obtained from Sigma-Aldrich (St. Louis, MO, USA). Ethanol (for UV-VIS spectroscopy, min. 99.8\%) was purchased from Penta (CZ). The chromatographic columns Rxi-5MS, Rxi-17Sil MS, Rtx-200 MS (Restek, Bellefonte, PA, USA) and TG-5HT (Thermo Scientific, Waltham, MA, USA) had the same column diameter of $0.25 \mathrm{~mm}$ and a stationary phase thickness of $0.25 \mu \mathrm{m}$. The carrier gas was helium 5.5 (purity $\geq 99.9995)$ from Linde $(\mathrm{CZ})$.

\subsection{Preparation of a Mixture of Standards}

To develop and optimize the chromatographic measurement method, a model mixture of human skin scent containing 98 compounds (see Supplementary Materials, Table S1) with a concentration of $100 \mathrm{mg} \cdot \mathrm{L}^{-1}$ of each component in hexane was created. A solution with a concentration of $25 \mathrm{mg} \cdot \mathrm{L}^{-1}$ of each component in hexane was used for the work. The compounds were chosen to cover the widest possible range (chemical family, polarity, molecular weight) of compounds found in human skin scent samples.

\subsection{Preparation of Real Scent Samples}

The developed chromatographic methods were also verified by measuring real scent samples. The scent samples were taken on glass beads of the palms of volunteers. Details on the purification of the sampling material and the scent sampling are given in Appendix A. The samples were subsequently extracted by double extraction into $1 \mathrm{~mL}$ of ethanol, each extraction step lasting $20 \mathrm{~min}$, of which $10 \mathrm{~min}$ extraction was supported by shaking (1000 rpm, IKAVIBRAX VXR basic, Staufen im Breisgau, Germany) and $10 \mathrm{~min}$ by placing the samples in an ultrasonic bath (Fisherbrand FB15059, Fisher Scientific, Loughborough, UK). The individual extracts were gradually evaporated to dryness in a $1.5 \mathrm{~mL}$ vial in a Genevac EZ-2 concentrator under reduced pressure (approx. 100 mbar). The residue was then redissolved in $100 \mu \mathrm{L}$ of a 1:1 mixture of ethanol and hexane. The samples prepared in this way were stored in a refrigerator at $7^{\circ} \mathrm{C}$ until the analysis.

\subsection{Instrumentation}

The samples were analyzed on a 7890B GC gas chromatograph (Agilent, Santa Clara, CA, USA) with a Pegasus ${ }^{\circledR}$ 4D-C time-of-life mass detector from LECO Corp. (St. Joseph, MI, USA). A MultiPurpose Sampler MPS Robotic from Gerstel (Mülheim, Germany) was used to dose samples to the column. The sample measurement and data evaluation were performed in the ChromaTOF ${ }^{\circledR}$ program (version 4.72.0.0, LECO Corp., St. Joseph, MI, USA).

During the development of the method, five main column configurations were tested, namely:

1. Classic arrangement: non-polar Rxi-5MS $(2+30 \mathrm{~m})+$ medium polar Rxi-17Sil MS (1 m);

2. Classic arrangement: non-polar Rxi-5MS $(2+30 \mathrm{~m})+$ medium polar Rtx-200MS $(1 \mathrm{~m})$;

3. Reverse arrangement: medium polar Rxi-17Sil MS $(2+30 \mathrm{~m})+$ non-polar TG-5HT (1 m);

4. Reverse arrangement: medium polar Rtx-200 MS (2+30 m) + non-polar TG-5HT (1 m);

5. Reverse arrangement: medium polar Rtx-200 MS $(2+15 \mathrm{~m})+$ non-polar TG-5HT $(1 \mathrm{~m})$,

where the first bracket shows the length of the pre-column and the primary column, the pre-column being the same as the primary column, and the second bracket shows the total length of the secondary column, of which $10 \mathrm{~cm}$ is in the modulator, $21 \mathrm{~cm}$ in the GC-MS transfer line and $69 \mathrm{~cm}$ in the secondary oven. 
The injection of samples $(1 \mu \mathrm{L})$ took place at a temperature of $280^{\circ} \mathrm{C}$ in the splitless mode with a period of $2 \mathrm{~min}$. The carrier gas flow was set at a constant $1.5 \mathrm{~mL} / \mathrm{min}$. The optimized temperature program was: $40^{\circ} \mathrm{C}-2 \mathrm{~min}-5^{\circ} \mathrm{C} / \mathrm{min}-320^{\circ} \mathrm{C}-10 \mathrm{~min}$, the total time of one analysis was $68 \mathrm{~min}(4080 \mathrm{~s})$. The secondary column was heated to a temperature $5{ }^{\circ} \mathrm{C}$ higher than the primary column and the modulator to a temperature $15^{\circ} \mathrm{C}$ higher than the secondary column. In the case of a $15 \mathrm{~m}$ primary column instead of $30 \mathrm{~m}$, the temperature program was shortened by $10 \mathrm{~min}$ (the final holding of the final temperature was eliminated), and the secondary column was heated $10{ }^{\circ} \mathrm{C}$ higher than the primary column.

Cryogenic modulation was performed on a secondary column using dried air at $-80^{\circ} \mathrm{C}$. The modulation periods and the corresponding hot and cold pulse times were different for different column configurations; see Table 1 . The warm pulse length corresponds to 0.3 times the modulation period length and the cold pulse length to 0.2 times the modulation period length.

Table 1. Modulation periods and durations of hot and cold pulses for different column configurations.

\begin{tabular}{ccccc}
\hline Column Configuration & Time [s] & Modulation Period [s] & Hot Pulse [s] & Cold Pulse [s] \\
\hline Rxi-5 ms/Rxi-17Sil MS & $500-4080$ & 4 & 1.2 & 0.8 \\
\hline Rxi-5 ms/Rtx-200 MS & $500-4080$ & 4 & 1.2 & 0.8 \\
\hline \multirow{2}{*}{ Rxi-17Sil MS/TG-5HT } & $500-2592$ & 4 & 1.8 & 0.8 \\
& $2592-3198$ & 6 & 2.4 & 1.2 \\
\hline & $3198-4080$ & 8 & 1.8 & 1.6 \\
\hline
\end{tabular}

The transfer line was set to $280^{\circ} \mathrm{C}$. The mass detector was in electron ionization (EI) mode with an ionizing electron energy of $70 \mathrm{eV}$, and the temperature of the ion source was $250{ }^{\circ} \mathrm{C}$. Data collection took place in the Total Ion Current (TIC) mode; in the range $29-800 \mathrm{~m} / z$, the data collection speed was $200 \mathrm{spectra} / \mathrm{s}$. The acquisition delay was set to $500 \mathrm{~s}$ for a $30 \mathrm{~m}$ primary column, and in the case of a $15 \mathrm{~m}$ column it was shortened to $300 \mathrm{~s}$. The voltage on the detector was set $200 \mathrm{~V}$ higher than tuning to increase the detection sensitivity.

\subsection{Choosing the Best System Configuration}

The different systems were compared with each other in terms of orthogonality, the used separation space, the maximum theoretical peak capacity and the condition peak capacity of the system, the achieved resolution, and the number of coelutions.

\subsubsection{Spearman's Correlation Coefficient}

Spearman's correlation coefficient is used to measure monotonic nonlinear correlations between retention times and can be used to measure the orthogonality of systems [16]. First, the order of retention times in the first $\left(r_{i}^{t 1}\right)$ and second dimensions $\left(r_{i}^{t 2}\right)$ is determined for compound $i$. The Spearman's correlation coefficient $(\rho)$ is then calculated according to Equation (1),

$$
\rho=1-\frac{6 \sum_{i=1}^{n} d_{i}^{2}}{n\left(n^{2}-1\right)}
$$

where $d_{i}=r_{i}^{t 1}-r_{i}^{t 2}$ and $n$ is the number of compounds. 


\subsubsection{Bin Counting Method}

The bin counting method $[17,18]$ serves both as an indicator of orthogonality and an indicator of the coverage of the separation space. In the bin counting method, retention times in both dimensions must first be normalized according to Equation (2),

$$
t_{r, \text { norm }(i)}=\frac{t_{r(i)}-t_{r, \text { first }}}{t_{r, \text { last }}-t_{r, \text { first }}}
$$

where ${ }^{1} t_{r, \text { norm(i) }}$ and ${ }^{2} t_{r, \text { norm(i) }}$ are normalized retention times in the first and the second dimension, $t_{r(i)}$ is the retention time of compound $i$, and $t_{r, f i r s t}$ and $t_{r, l a s t}$ are the retention times of the first and last observed peaks in the separation space, respectively.

Then, the separation space is divided into bins, whose number is the same or very close to the number of compounds in the sample. Subsequently, the number of bins containing a peak is counted and compared with the total number of bins according to Equation (3),

$$
O=\frac{\sum \text { bins }-\sqrt{P_{\max }}}{0.63 P_{\max }}
$$

where $P_{\max }$ is the total number of bins. Occupied space $O$ takes values from 0 (completely non-orthogonal system) to 1 (completely orthogonal system). The coefficient of 0.63 is based on the observation that systems close to orthogonality have a ratio of filled and total bins (so-called $f_{\text {coverage }}$ ) close to 0.63 .

For reverse systems, the separation space was divided into three parts, which corresponded to the individual modulation periods. The total value $O$ of the given reverse system was calculated as a weighted average of partial $O$.

\subsubsection{Peak Capacities}

The theoretical peak capacities [19] of the system in both dimensions $\left({ }^{1} n_{c}\right.$ and $\left.{ }^{2} n_{c}\right)$ were calculated according to Equation (4).

$$
n_{c}=\frac{t_{r, l a s t}-t_{r, \text { first }}}{w_{b}}
$$

where $t_{r}$ is the retention time of the last of first observed peaks in the separation space, and $w_{b}$ is the measured average $4 \sigma$ peak width. The maximum theoretical GC $\times$ GC capacity of the ${ }^{2 D} n_{c}$ system was then determined as the product of the peak capacities in the first $\left({ }^{1} n_{c}\right)$ and the second $\left({ }^{2} n_{c}\right)$ dimensions (Equation (5)).

$$
{ }^{2 D} n_{c}={ }^{1} n_{c} \cdot{ }^{2} n_{c}
$$

However, orthogonality and surface coverage have a huge impact on the calculations of the conditional (practical) peak capacity ${ }^{2 D} n_{c, \text { cond }}$ of a comprehensive $2 \mathrm{D}$ system [19]. It is given by Equation (6),

$$
{ }^{2 D} n_{c, \text { cond }}={ }^{1} n_{c} \cdot{ }^{2} n_{c} \cdot f_{\text {coverage }} \cdot \frac{1}{\beta}
$$

where $f_{\text {coverage }}$ is the ratio of filled and total bins (see Section 2.5.2), and $\beta$ is an undersampling of the first dimension, and it is given by Equation (7),

$$
\beta=\sqrt{1+0.21\left(\frac{t_{s}}{1_{\sigma}}\right)^{2}}
$$

where $t_{s}$ is the first-dimension sampling time, and ${ }^{1} \sigma$ is the peak standard deviation in the first dimension.

For reverse systems, the separation space was divided into three parts, which corresponded to the individual modulation periods. The total value of theoretical peak capacity 
and the conditional peak capacity of the given reverse system were calculated as a sum of partial capacitances.

\subsubsection{Resolutions}

With knowledge of the maximum theoretical peak capacities (see Equation (4)), the average resolutions $\left(R_{s, a v}\right)$ in both dimensions can be calculated [20]. It is given by Equation (8),

$$
R_{s, a v}=\frac{n_{c}}{n-1}
$$

where $n$ is the number of compounds.

The resolutions of the two adjacent peaks in the individual dimensions $\left({ }^{1} R s\right.$ and $\left.{ }^{2} R s\right)$ were calculated from the retention times $\left(t_{r 1}\right.$ and $\left.t_{r 2}\right)$ and the width of the peaks at the base $\left(w_{b 1}\right.$ and $\left.w_{b 2}\right)$ according to Equation (9) [21],

$$
R s=\frac{t_{r 2}-t_{r 1}}{\left(w_{b 1}+w_{b 2}\right) / 2}
$$

and the total resolution in a $2 \mathrm{D}$ system of ${ }^{2 D} R s$ was then calculated as the square root of the sum of the squares of the resolutions obtained in the first and second dimensions; see Equation (10) [21].

$$
{ }^{2 D} R s=\sqrt{{ }^{1} R s^{2}+{ }^{2} R s^{2}}
$$

\section{Results}

3.1. Classic Arrangement: Non-Polar Rxi-5MS $(2+30 \mathrm{~m})+$ Medium Polar Rxi-17Sil MS (1 m)

The first column arrangement tested was the $30 \mathrm{~m}$ Rxi-5ms column with the $2 \mathrm{~m}$ Rxi-5ms precolumn in the first dimension and the $1.5 \mathrm{~m}$ Rxi-17Sil MS column in the second dimension. The Rxi-5ms column with a (5\%-phenyl)-methylpolysiloxane phase has a low polarity, which is suitable for a wide range of analyses. The second Rxi-17Sil MS column with a (50\%-phenyl)-methylpolysiloxane phase is characterized by medium polarity and higher selectivity to aromatic substances and is usually recommended for environmental analyses. After the initial analyses, however, it was observed that when using a column length of $1.5 \mathrm{~m}$ in the second dimension, the retention times in the second dimension are too high related to the modulation period and a large area of unused separation space was formed at the bottom of the chromatogram. For this reason, the secondary column was shortened to $1 \mathrm{~m}$, and this column length was used as the standard for the other column arrangements.

The ramp of the temperature program was also tested under the above conditions (see Section 2.4). At a temperature rate of $3{ }^{\circ} \mathrm{C} / \mathrm{min}$, the temperature program was very long $(105 \mathrm{~min})$, at $8^{\circ} \mathrm{C} / \mathrm{min}$, the temperature program was shortened to $47 \mathrm{~min}$, however, the resolution of the peaks in the first dimension deteriorated significantly. The rate of $5{ }^{\circ} \mathrm{C} / \mathrm{min}$ was chosen as the most suitable, as a compromise between the total length of the program and the achieved resolution in the first dimension.

Figure 1 shows the chromatogram obtained under the optimized conditions (see Section 2.4). The classification zones of homologues of various compounds are highlighted. $n$-alkanes are marked with black contour, with which amines partially coelute (lower dark yellow zone). For instance, heptadecane with $\mathrm{N}, \mathrm{N}$-dimethyltetradecanamide achieved an overall resolution of ${ }^{2 D} R s=0.65$. Acids and esters (left red zone), aldehydes and ketones (yellow zone), alcohols (left white zone), and chloroalkanes (green zone) showed similar retention on the secondary column. For example, dodecan-1-ol with undecanoic acid achieved an overall resolution of ${ }^{2 D} R s=0.52$ and dodecan-1-ol with 1-chlordodecane ${ }^{2 D} R s$ $=0.56$. Ethers (right white zone) and amides (upper dark yellow zone) are then clearly separated from these compounds. The right red zone then represents the area of higher fatty acid esters. Unlabeled compounds with higher retention on the secondary column represent compounds classified as "others", which are often part of cosmetic products. 
The list of standards including retention indices calculated from absolute retention times according to [22] is given in Supplementary Material, Table S1.

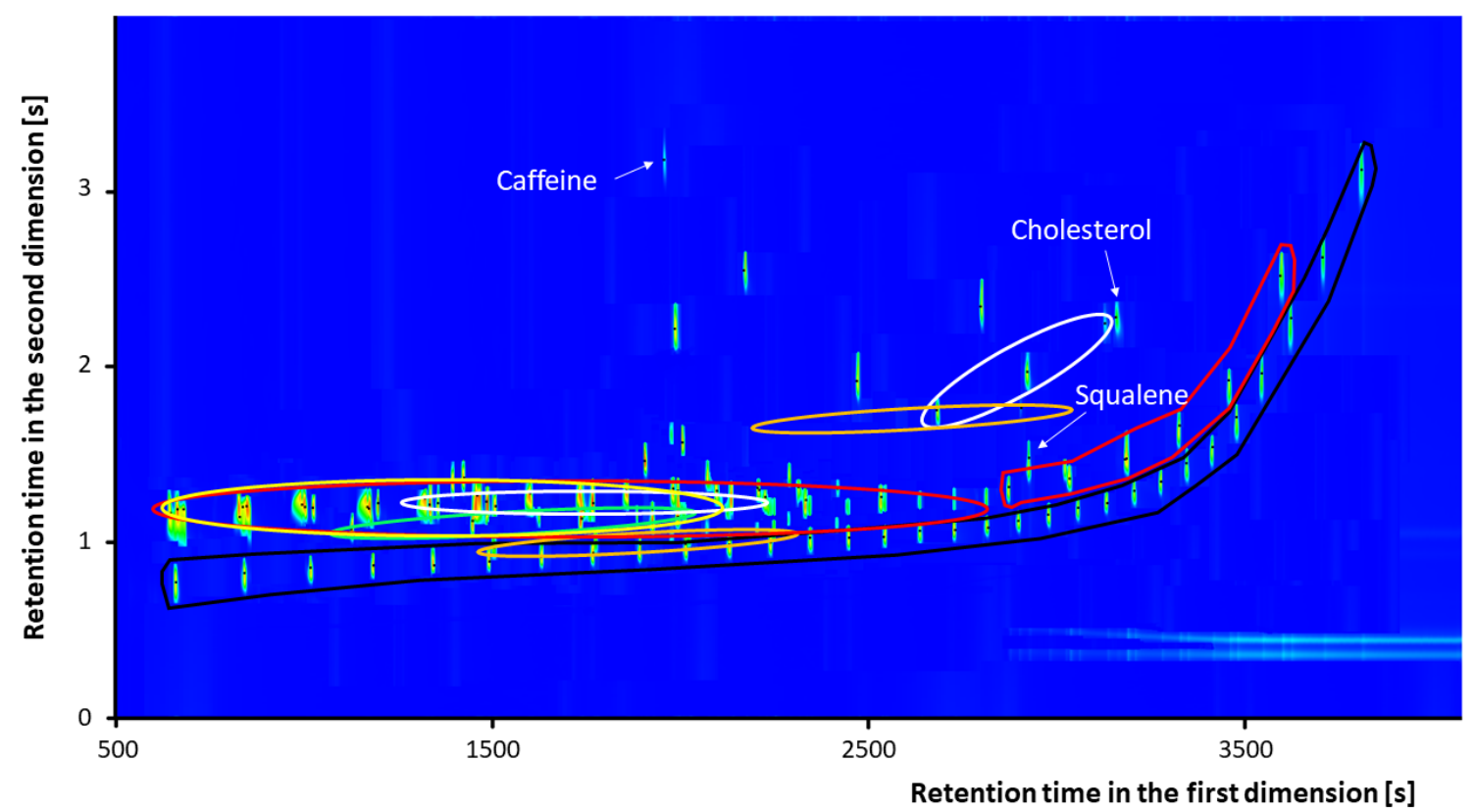

Figure 1. The TIC 2D chromatogram of the model mixture (composition see Table 1) measured under the conditions described in Section 2.4 on Rxi-5ms $(2+30 \mathrm{~m})$ and Rxi-17Sil MS $(1 \mathrm{~m})$ columns. The marked classification zones are the following: black ( $n$-alkanes), lower dark yellow (amines), upper dark yellow (amides), green (chloroalkanes), left white (alcohols), right white (ethers), yellow (aldehydes and ketones), left red (acids and esters), right red (esters of higher fatty acids), and unlabeled (others).

Unfortunately, it was not possible to distinguish structural isomers such as hexadecyl ester of tetradecanoic acid and dodecyl ester of octadecanoic acid in the model mixture $\left({ }^{2 D} R s=0\right.$, both compounds have the general formula $\mathrm{C}_{30} \mathrm{H}_{60} \mathrm{O}_{2}$ and $\mathrm{M}_{\mathrm{r}}=452.80$, their mixed mass spectrum see Figure 2). The total number of analyte coelutions (with ${ }^{2 D} R s<1.5$ ) in the mixture was 38 .

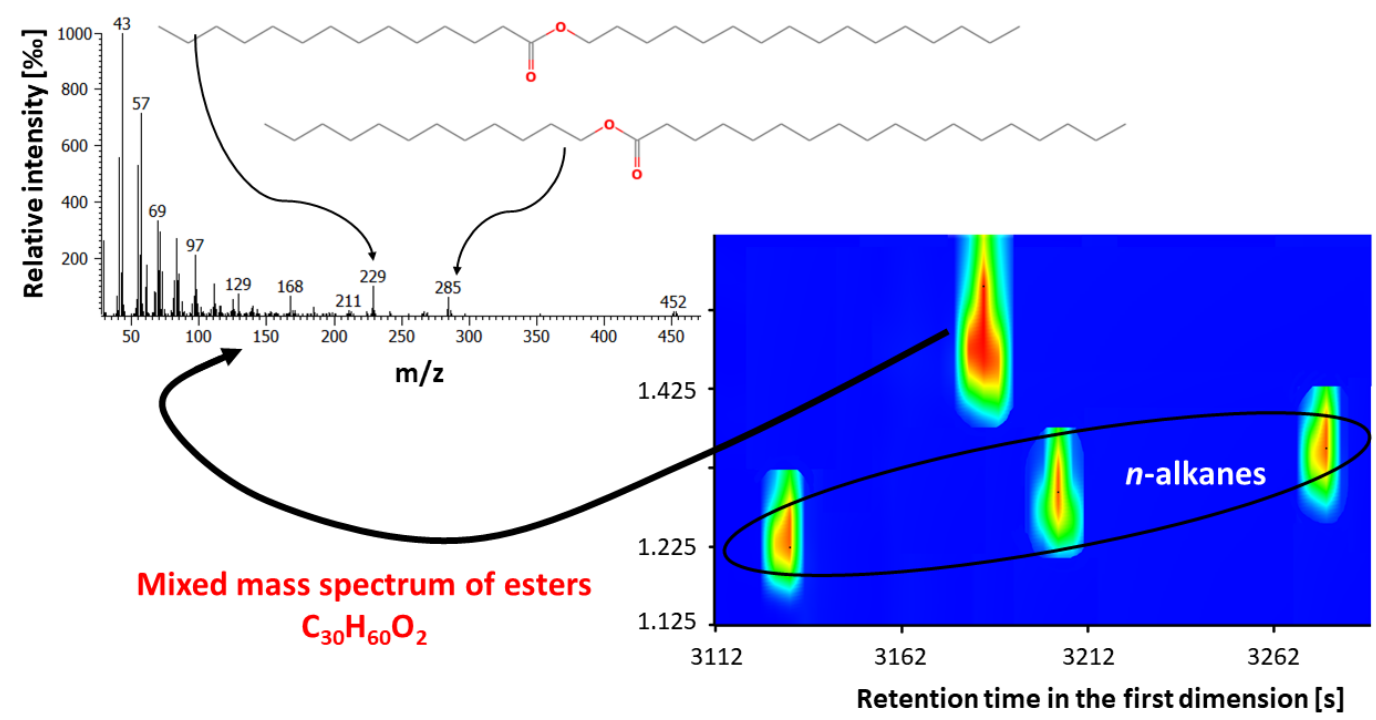

Figure 2. The mixed mass spectrum of hexadecyl ester of tetradecanoic acid and dodecyl ester of octadecanoic acid. The arrows indicate their typical $\mathrm{m} / \mathrm{z}$, which correspond to the loss of the alkyl chain of the alcohol associated with the migration of two hydrogens. 
As described above, the Rxi-17Sil MS column is selective for aromatics, resulting in high retention of aromatic compounds in the second dimension. This was also shown in the subsequent analysis of the real sample, when the modulation period of $4 \mathrm{~s}$ was too short, and the so-called wrap-around effect occurred; see Figure 3.

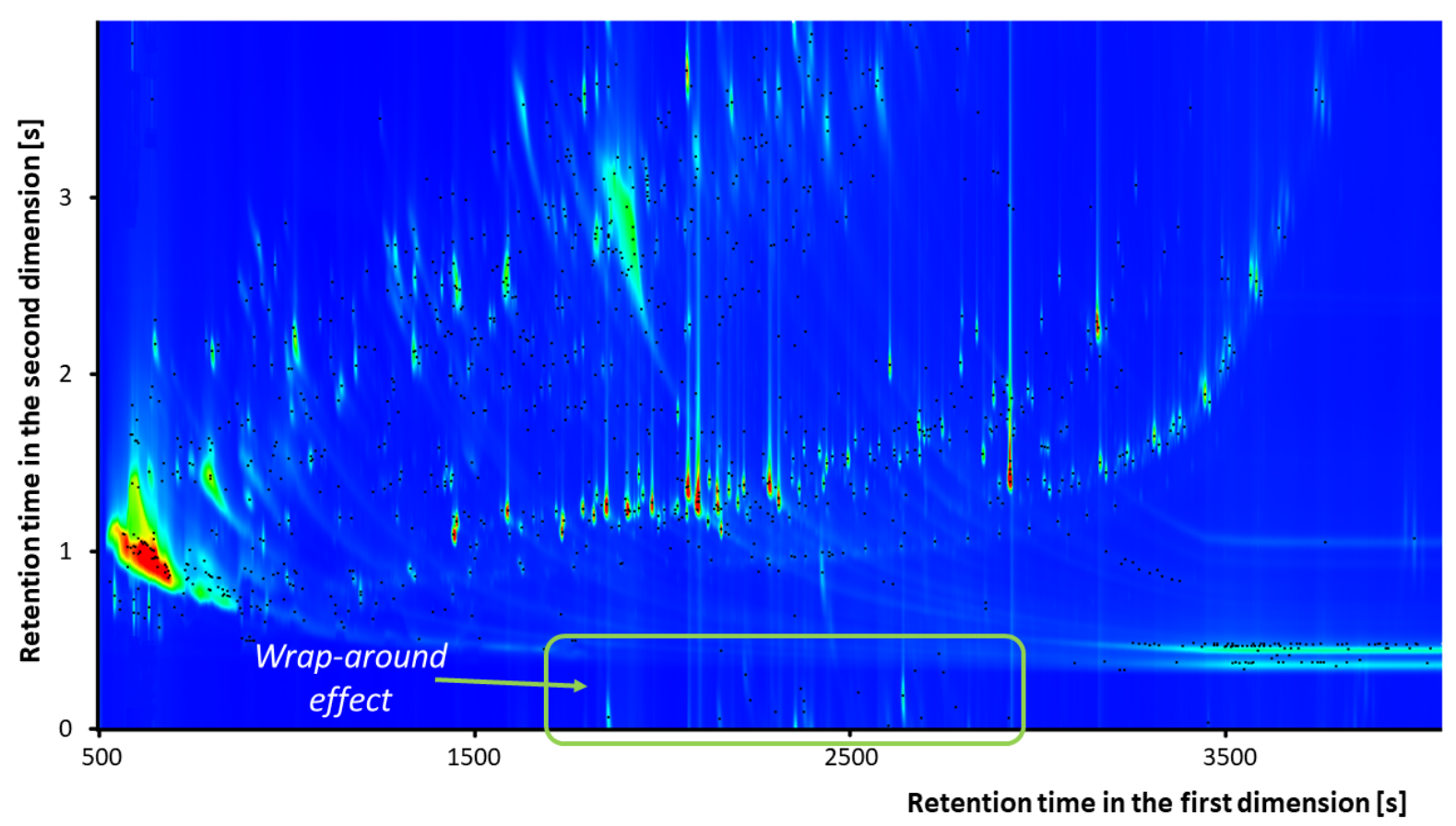

Figure 3. The TIC 2D chromatogram of a real scent sample measured under the conditions described in Section 2.4 on Rxi-5ms $(2+30 \mathrm{~m})$ and Rxi-17Sil MS (1 m) columns.

\subsection{Classic Arrangement: Non-Polar Rxi-5MS $(2+30 \mathrm{~m})+$ Medium Polar Rtx-200MS (1 m)}

In another tested arrangement, the secondary column was replaced with the Rtx200MS column with a (trifluoropropyl)-methylpolysiloxane phase, which, like Rxi-17Sil MS, belongs to the group of the mid-polar columns; however, the polarity is slightly higher. The great advantage of the Rtx-200MS column is its unique selectivity for electronegative substances, which changes the elution order and can ensure a resolution that cannot be achieved on the phenyl, cyano or polyethylene glycol phases.

The chromatogram of the model mixture measured on the Rxi-5ms and the Rtx-200MS columns under optimized conditions (see Section 2.4) is then shown in Figure 4. Several differences can be observed compared to the chromatogram in Figure 1. First, there was a clear separation of aldehydes and ketones in the second dimension (yellow classification zone) from acids and esters (first red zone). Second, there was a significant reduction in aromatic retention in the second dimension (see caffeine, which had ${ }^{2} \mathrm{tr}=3.18 \mathrm{~s}$ for the Rxi-17Sil MS column, ${ }^{2} \operatorname{tr}=1.66$ s for the Rtx-200 MS column). However, the worstseparated places remained poorly differentiated. Amines (lower dark yellow zone) still coeluted with $n$-alkanes (black zone), e.g., the previously discussed heptadecane with $\mathrm{N}, \mathrm{N}$-dimethyltetradecanamine reached ${ }^{2 D} R s=0.57$. Alcohols (left white zone) then still showed similar retention as acids (left red zone) and chloroalkanes (green zone). For example, dodecan-1-ol with undecanoic acid achieved an overall resolution of ${ }^{2 D} R s=0.65$ and dodecan-1-ol with 1-chlordodecane ${ }^{2 D} R s=0.61$. The structural isomers in the area of higher fatty acid esters (right red zone) were not distinguished at all $\left({ }^{2 D} R s=0\right)$. The total number of analyte coelutions (with ${ }^{2 D} R s<1.5$ ) in the mixture was 34 . 


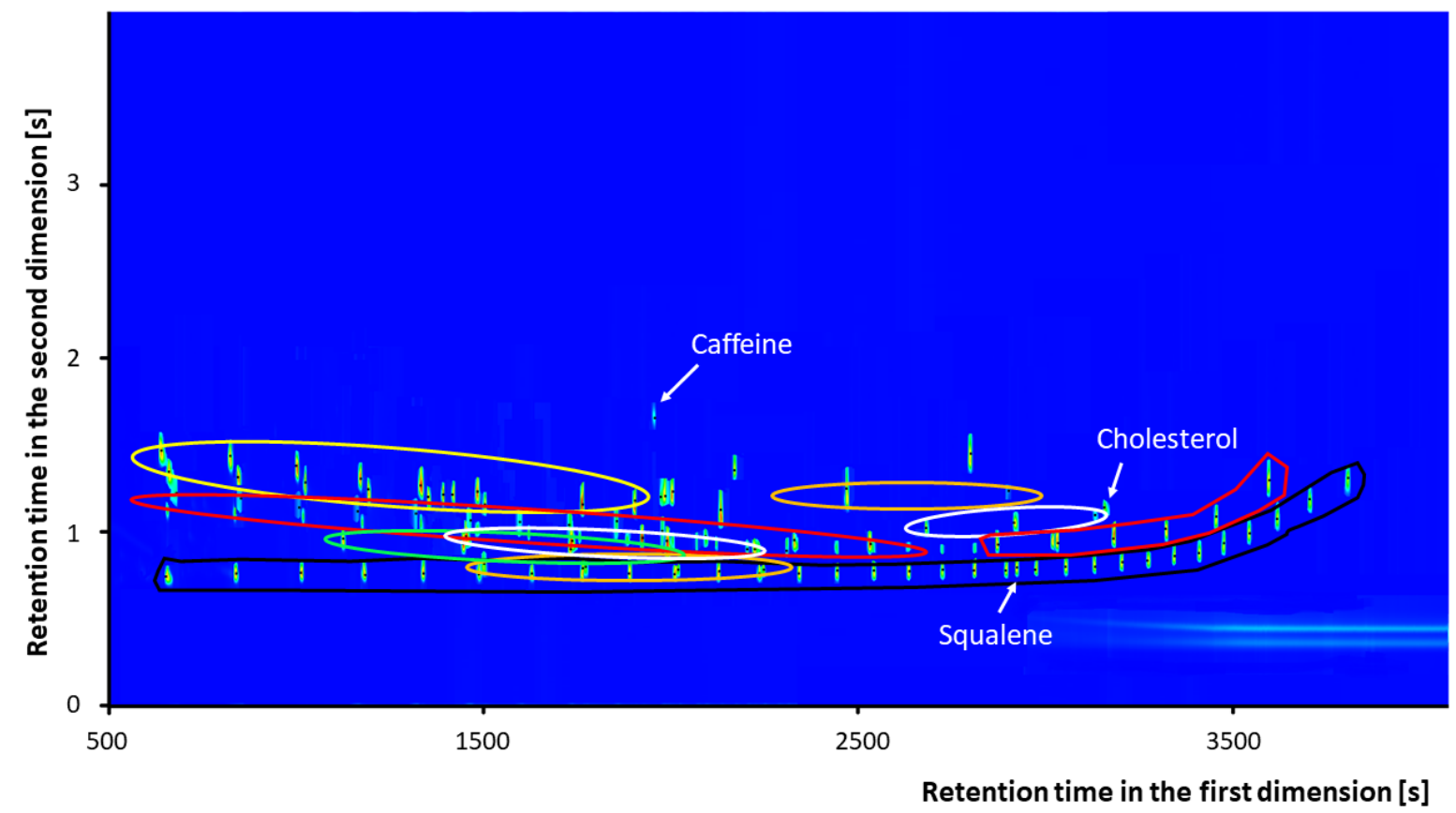

Figure 4. The TIC 2D chromatogram of the model mixture (composition see Table 1) measured under the conditions described in Section 2.4 on Rxi-5ms $(2+30 \mathrm{~m})$ and Rxi-200MS $(1 \mathrm{~m})$ columns. The marked classification zones are the following: black ( $n$-alkanes), lower dark yellow (amines), upper dark yellow (amides), green (chloroalkanes), left white (alcohols), right white (ethers), yellow (aldehydes and ketones), left red (acids and esters), right red (esters of higher fatty acids), and unlabeled (others).

Due to the lower retention of aromatic compounds, in the case of a real sample, there was no longer a wrap-around effect during the modulation period of $4 \mathrm{~s}$. See Figure 5 for the chromatogram of the real sample.

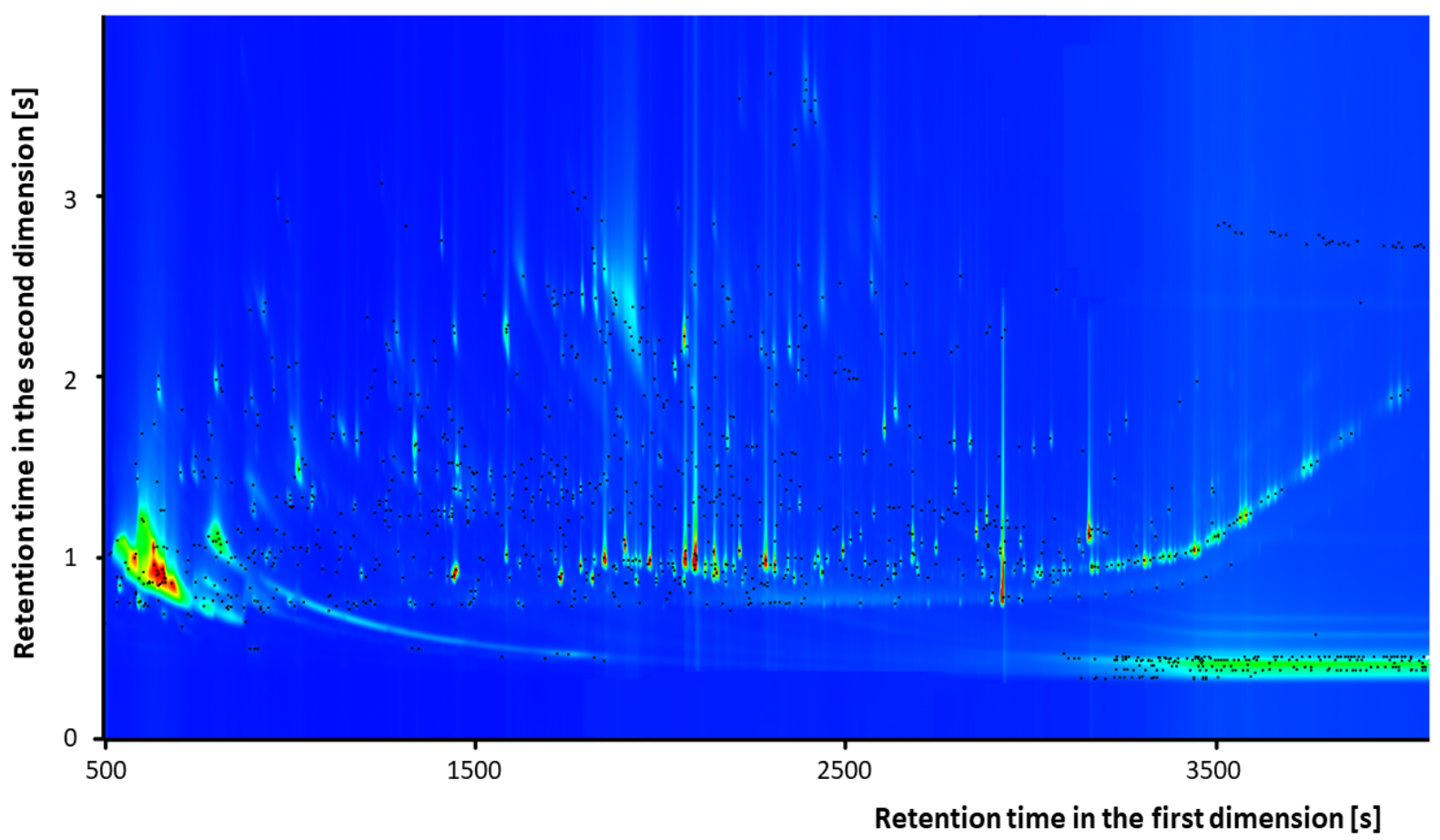

Figure 5. The TIC 2D chromatogram of a real scent sample measured under the conditions described in Section 2.4 on Rxi-5ms (2+30 m) and Rtx-200MS (1 m) columns. 


\subsection{Reverse Arrangement: Medium Polar Rxi-17Sil MS $(2+30 \mathrm{~m})+$ Non-Polar TG-5HT $(1 \mathrm{~m})$}

Due to the failure of achieving satisfactory separation in the classical arrangement of the columns, the order of the tested columns was reversed. The first reverse system consisted of the $30 \mathrm{~m}$ primary column Rxi-17Sil MS with the $2 \mathrm{~m}$ precolumn, also Rxi-17Sil MS. A $1 \mathrm{~m}$ long TG-5HT was chosen as the secondary column, which is formed as the Rxi-5ms by a non-polar (5\%-phenyl)-methylpolysiloxane phase; however, it is designed up to temperatures of $400{ }^{\circ} \mathrm{C}$ and the manufacturer guarantees up to $40 \%$ longer life compared to similar columns.

Figure 6 shows the measured chromatogram obtained under optimal conditions (see Section 2.4), from which it is clear, that the elution of different types of substances was reversed compared to the classical systems. The highest retention on the secondary column had $n$-alkanes (black classification zone), from which amines (upper dark yellow zone) were visibly separated, e.g., the mentioned problem site of heptadecane and N,Ndimethyltetradecanamine reached an overall resolution of ${ }^{2 D} R s=4.15$. Acids and esters (left red zone) and aldehydes and ketones (yellow zone) were separated from each other within the first dimension, but not within the second dimension. Alcohols (left white zone), such as dodecan-1-ol, were further separated from the acids in the first dimension, and undecanoic acid reached a resolution of ${ }^{2 D} R s=1.15$. Previously mentioned problematic places, namely, alcohols (white zone) and chloroalkanes (green zone), were successfully separated within the first and second dimensions (the distinction between dodecan-1-ol and 1-chlordodecane reached ${ }^{2 D} R s=3.51$ ). The structural isomers in the area of higher fatty acid esters (right red zone) again remained indistinguishable $\left({ }^{2 D} R s=0\right)$. However, the distribution of amines and chloroalkanes deteriorated, e.g., for $\mathrm{N}, \mathrm{N}$-dimethyldodecanamine with 1-chlordodecane the resolution was ${ }^{2 D} R s=0.71$ (in both classical column arrangements the resolutions were ${ }^{2 D}$ Rs 3.7). The total number of analyte coelutions (with ${ }^{2 D} R s<1.5$ ) in the mixture was 24 . The chromatogram of a real scent sample is shown in Figure 7.

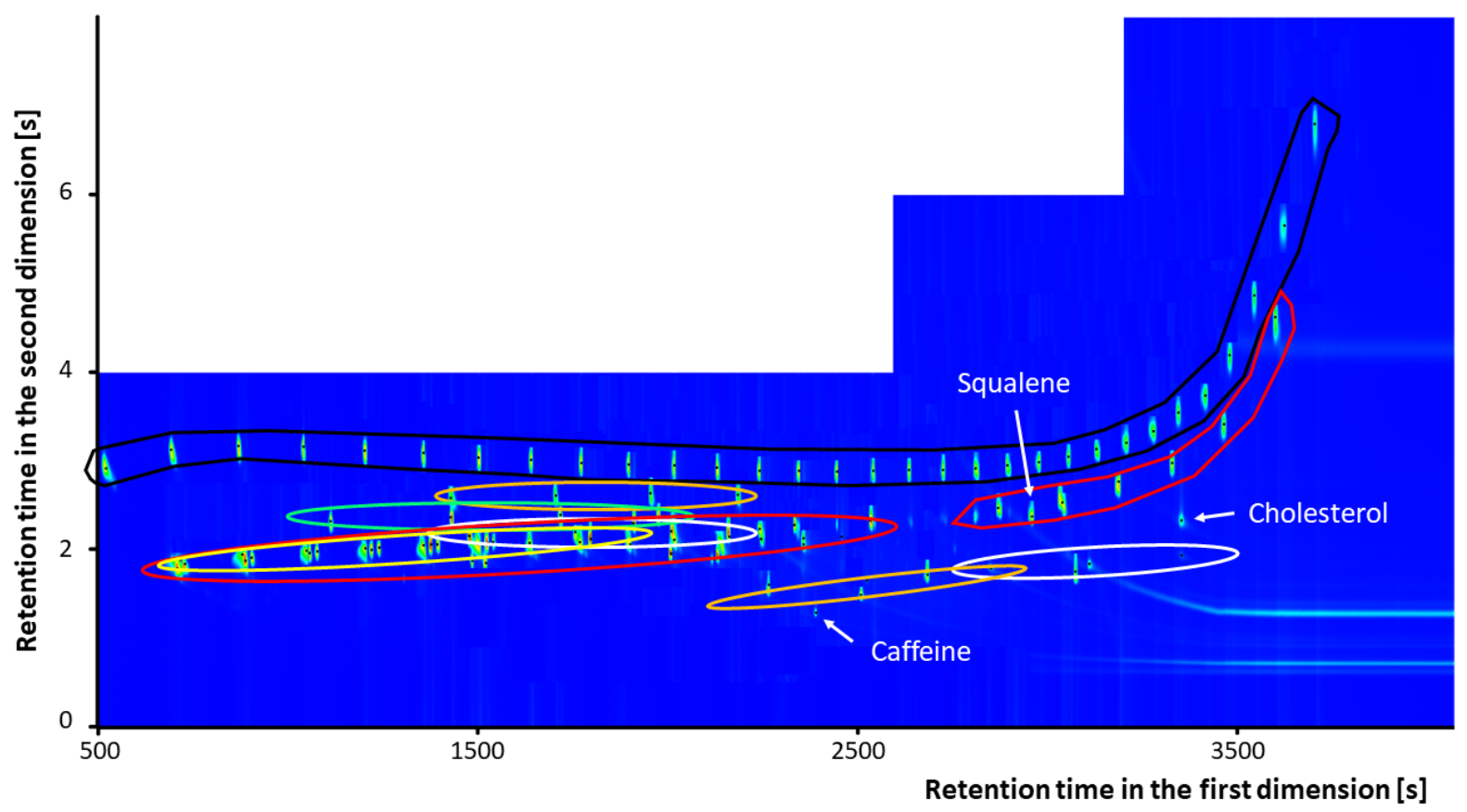

Figure 6. The TIC 2D chromatogram of the model mixture (composition see Table 1) measured under the conditions described in Section 2.4 on Rxi-17Sil MS $(2+30 \mathrm{~m})$ and TG-5HT $(1 \mathrm{~m})$ columns. The marked classification zones are the following: black ( $n$-alkanes), lower dark yellow (amines), upper dark yellow (amides), green (chloroalkanes), left white (alcohols), right white (ethers), yellow (aldehydes and ketones), left red (acids and esters), right red (esters of higher fatty acids), and unlabeled (others). 


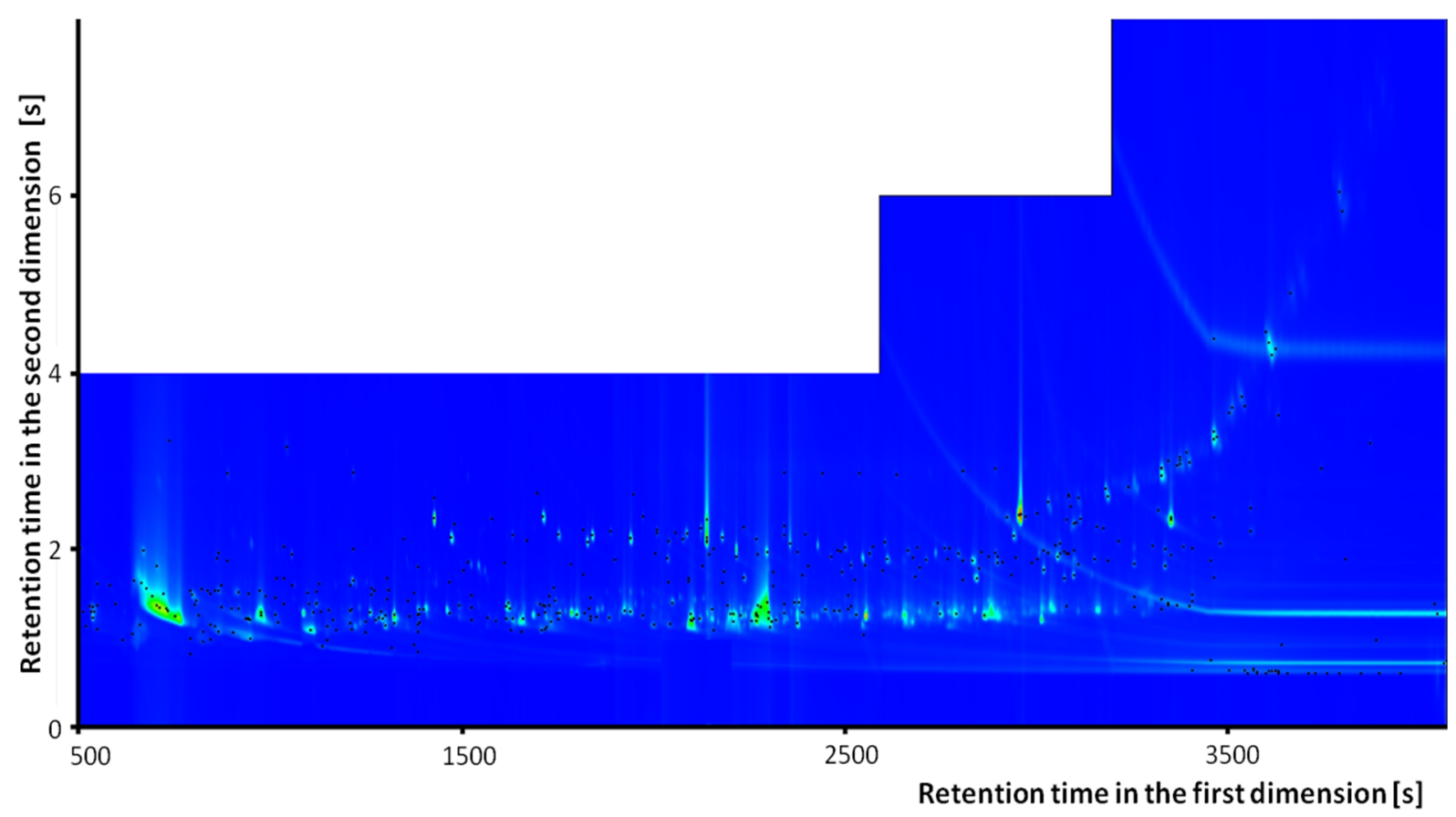

Figure 7. The TIC 2D chromatogram of a real scent sample measured under the conditions described in Section 2.4 on Rxi-17Sil MS (2+30 m) and TG-5HT (1 m) columns.

\subsection{Reverse Arrangement: Medium Polar Rtx-200 MS (2 + 30 m) + Non-Polar TG-5HT (1 m)}

The second reverse system consisted of the $30 \mathrm{~m}$ primary column Rtx-200 MS with the $2 \mathrm{~m}$ precolumn, also Rtx-200 MS. The $1 \mathrm{~m}$ long TG-5HT remained as a secondary column. Figure 8 shows that all studied compounds' groups have already been separated. In the second dimension, there was a more significant separation of amines (upper dark yellow classification zone) from chloroalkanes (green zone) within the second dimension, alcohols (left white zone) were more significantly separated from the area of acids and esters (left red zone), from whose zone also separated aldehydes and ketones (yellow zone). At the same time, however, there was a change in the retention of aromatic or polyunsaturated hydrocarbons (see squalene, cholesterol, caffeine). In this arrangement, squalene eluted near octacosane with a resolution of ${ }^{2 D} R s=1.34$ and cholesterol near tetradecyl ester of hexadecanoic acid $\left({ }^{2 D} R s=2.15\right)$. It was again not possible to distinguish the structural isomers in the region of higher fatty acid esters (second red bubble, ${ }^{2 D} R_{s}=0$ ). The total number of analyte coelutions (with ${ }^{2 D} R s<1.5$ ) in the mixture was 7 . The chromatogram of a real scent sample is shown in Figure 9. 


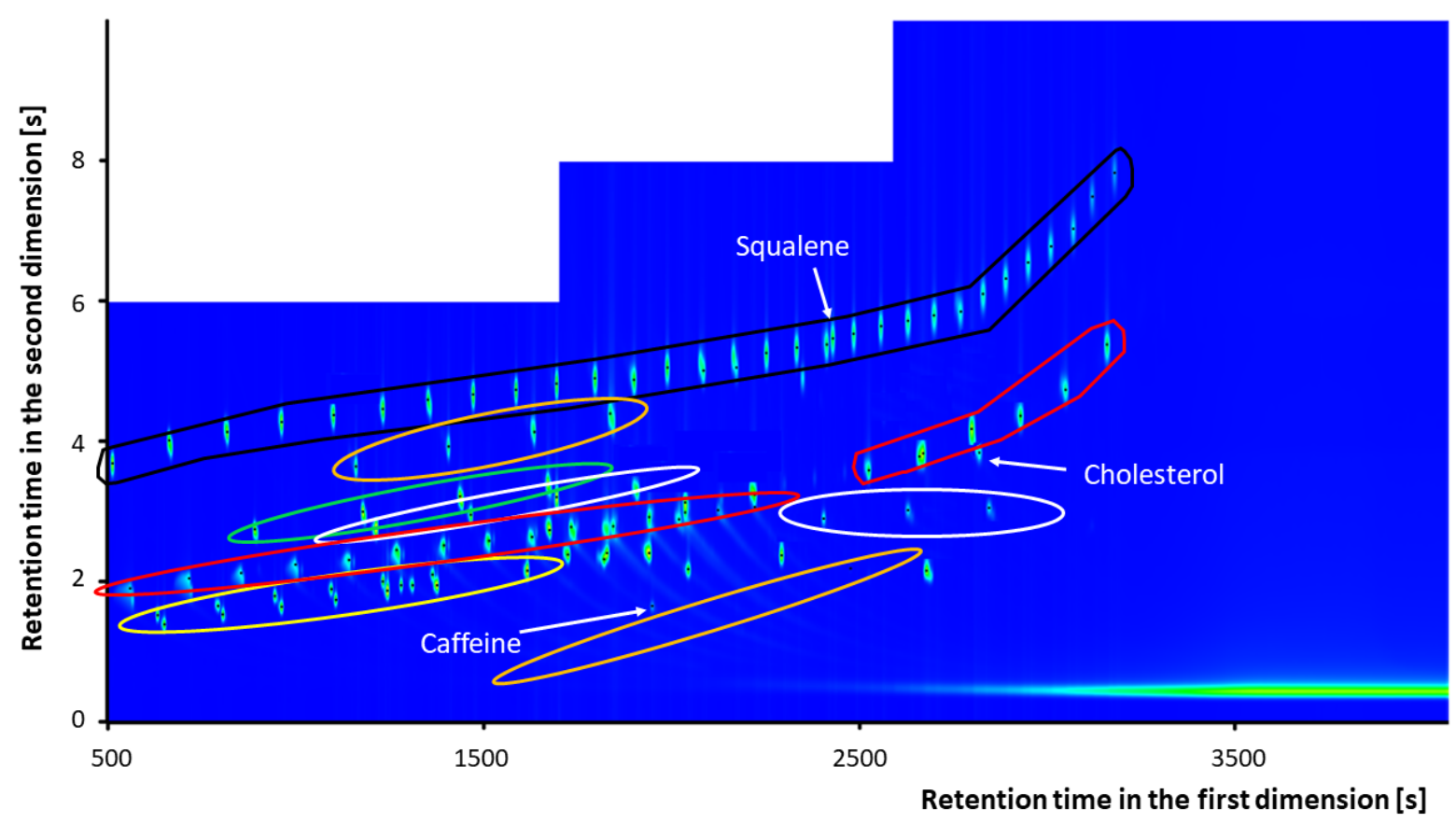

Figure 8. The TIC 2D chromatogram of the model mixture (composition see Table 1) measured under the conditions described in Section 2.4 on Rxi-200 MS $(2+30 \mathrm{~m})$ and TG-5HT $(1 \mathrm{~m})$ columns. The marked classification zones are the following: black ( $n$-alkanes), lower dark yellow (amines), upper dark yellow (amides), green (chloroalkanes), left white (alcohols), right white (ethers), yellow (aldehydes and ketones), left red (acids and esters), right red (esters of higher fatty acids), and unlabeled (others).

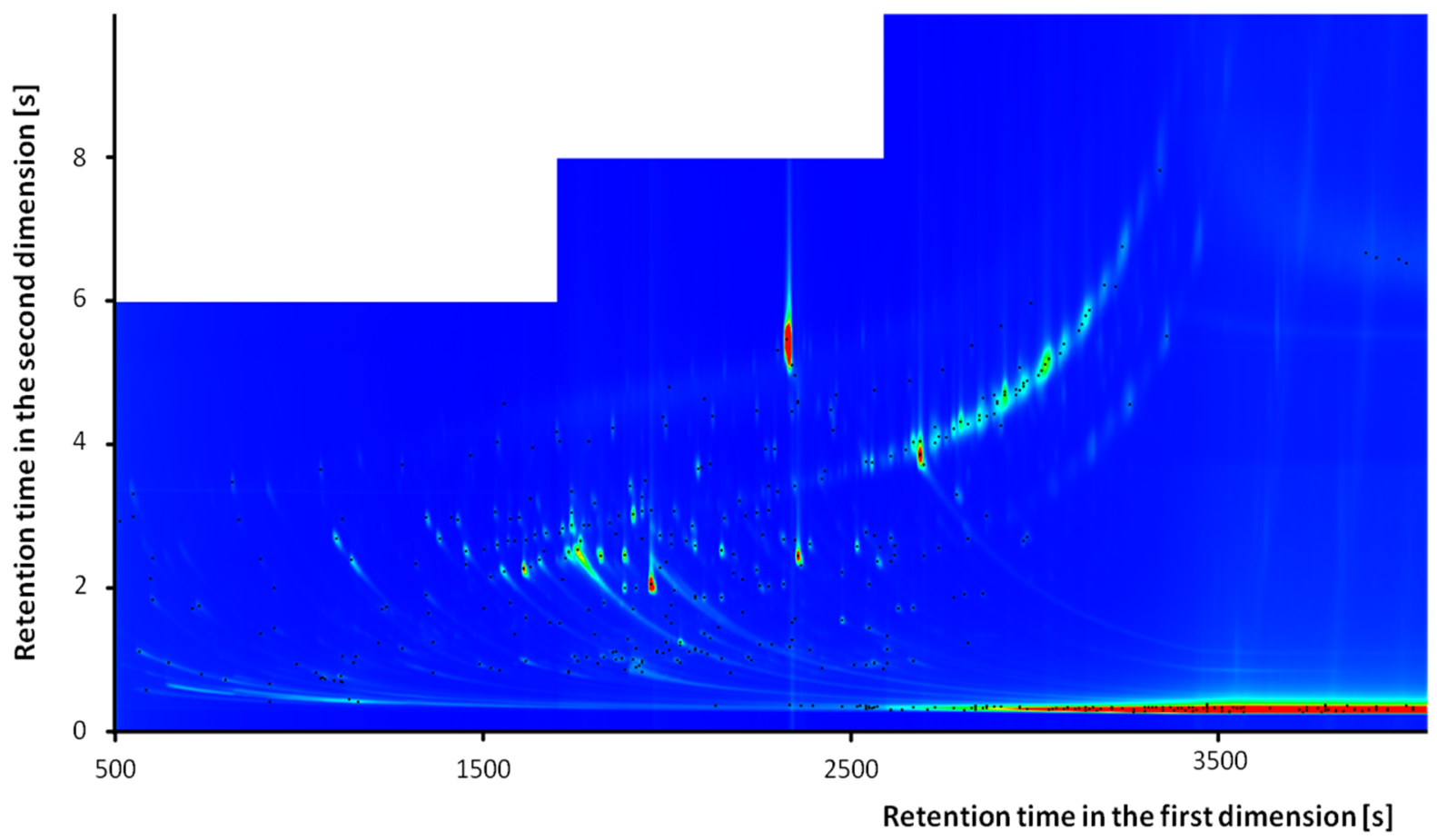

Figure 9. The TIC 2D chromatogram of a real scent sample measured under the conditions described in Section 2.4 on Rxi-200 MS (2+30 m) and TG-5HT (1 m) columns. 


\subsection{Reverse Arrangement 3: Medium Polar Rtx-200 MS $(2+15 \mathrm{~m})+$ Non-Polar TG-5HT $(1 \mathrm{~m})$}

To shorten the analysis time, an alternative to a $15 \mathrm{~m}$ primary column instead of a $30 \mathrm{~m}$ column was tested. While maintaining the original temperature gradient, the total analysis time was reduced by $10 \mathrm{~min}$ (for more details on setting up the analysis, see Section 2.4). In this configuration, however, the resolution of the compounds within the first dimension deteriorated (compared to the $30 \mathrm{~m}$ system, see Table 2) and the shape of the peaks deteriorated, in particular, their profile (see Figure 10). The total number of analyte coelutions (with ${ }^{2 D} R s<1.5$ ) in the mixture was 21 .

Table 2. The evaluation of orthogonality, use of separation space, peak capacities, average resolutions of individual systems, and the number of coelutions.

\begin{tabular}{|c|c|c|c|c|c|}
\hline Indicator & $\begin{array}{c}\text { Rxi-5 } \\
\text { ms/Rxi-17Sil MS }\end{array}$ & $\begin{array}{c}\text { Rxi-5 ms/Rtx-200 } \\
\text { MS }\end{array}$ & $\begin{array}{c}\text { Rxi-17Sil } \\
\text { MS/TG-5HT }\end{array}$ & $\begin{array}{c}\text { Rtx-200 MS (30 } \\
\text { m)/TG-5HT }\end{array}$ & $\begin{array}{c}\text { Rtx-200 MS (15 } \\
\text { m)/TG-5HT }\end{array}$ \\
\hline$\rho$ & 0.535 & -0.194 & 0.356 & 0.618 & 0.648 \\
\hline$O$ & 0.508 & 0.730 & 0.746 & 0.817 & 0.885 \\
\hline${ }^{2 D} n_{\mathrm{c}}$ & 2236 & 888 & 1896 & 3131 & 1474 \\
\hline $2 D_{n_{c, \text { cond }}}$ & 805 & 425 & 914 & 1329 & 589 \\
\hline${ }^{1} R_{S, a v}$ & 2.624 & 2.703 & 2.592 & 2.417 & 1.334 \\
\hline${ }^{2} R_{s, a v}$ & 0.087 & 0.034 & 0.273 & 0.432 & 0.324 \\
\hline${ }^{2 D} R_{S, a v}$ & 2.625 & 2.703 & 2.607 & 2.455 & 1.373 \\
\hline${ }^{2 D} R_{S}<1.5$ & 38 & 34 & 24 & 7 & 21 \\
\hline
\end{tabular}

$\rho$ is Spearman's correlation coefficient, $O$ is the occupied space, ${ }^{2 D} n_{c}$ is the theoretical maximal peak capacity, ${ }^{2 D} n_{c, \text { cond }}$ is the conditional peak capacity, $R_{s, a v}$ are resolutions in the first, second dimension or total $2 \mathrm{D}$ resolution, and ${ }^{2 D} R_{S}<1.5$ is the number of analyte pairs with ${ }^{2 D} R_{\mathrm{s}}<1.5$

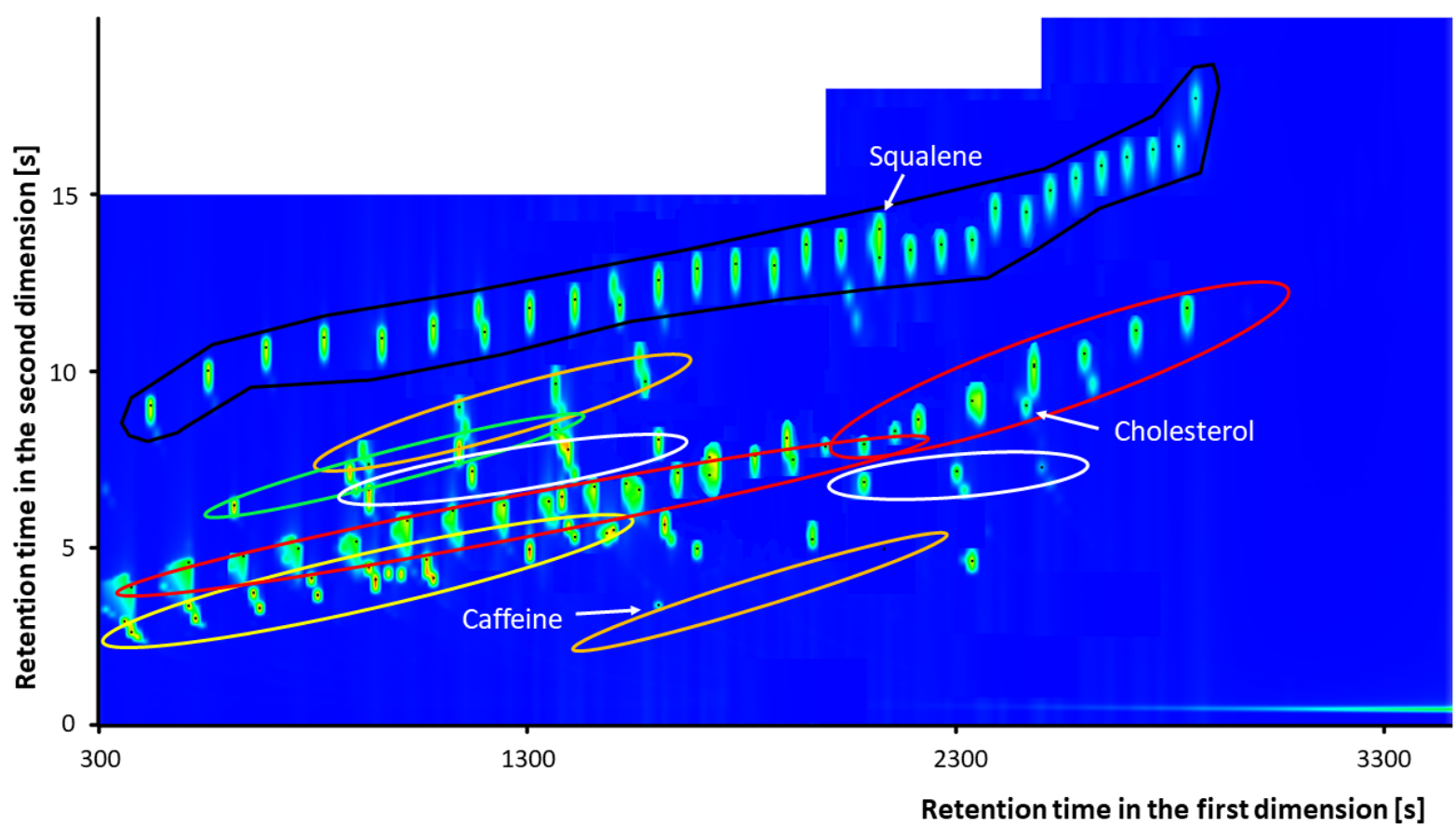

Figure 10. The TIC 2D chromatogram of the model mixture (composition see Table 1) measured under the conditions described in Section 2.4 on Rxi-200 MS $(2+15 \mathrm{~m})$ and TG-5HT $(1 \mathrm{~m})$ columns. The marked classification zones are the following: black ( $n$-alkanes), lower dark yellow (amines), upper dark yellow (amides), green (chloroalkanes), left white (alcohols), right white (ethers), yellow (aldehydes and ketones), left red (acids and esters), right red (esters of higher fatty acids), and unlabeled (others). 


\section{Discussion}

Five different configurations of the 2D chromatographic system were tested to find its optimal arrangement and at the same time with the intention of finding a suitable chromatographic method for non-targeted analysis of human odor samples. In testing chromatographic methods, mainly the temperature ramp was optimized. The rate of $5{ }^{\circ} \mathrm{C} / \mathrm{min}$ was chosen as the most suitable, as a compromise between the total length of the program and the achieved resolution in the first dimension. Individual chromatographic systems were evaluated in terms of orthogonality, use of separation space, peak capacities, resolutions, and finally the number of coelutions (see Table 2). The first of the tested systems in the classical arrangement (Rxi-5 ms/Rxi-17Sil MS) reached a high theoretical peak capacity; however, the use of separation space was the worst compared to the other tested systems. Thanks to this, the conditional peak capacity was considerably lower, and the number of coelutions of analytes of the model mixture was the highest (38). In the case of the analysis of a real scent sample, which contained, e.g., many aromatic heterocyclic compounds with high retention, the so-called wrap-around effect. The second system in the classical arrangement (Rxi-5ms/Rtx-200 MS) showed the highest orthogonality according to Spearman's correlation coefficient, and the use of the separation space was considerably higher than in the first case. The theoretical and conditional peak capacities were generally lower than in the first case, as the peak capacity in the second dimension decreased due to the lower retention of aromatic compounds (e.g., caffeine). However, the average resolution of the analytes improved slightly, but multiple coelutions still occurred (34 in total). Due to the lower retention of aromatic compounds, there was no longer a wrap-around effect in the analysis of a real scent sample. Reverse systems (except Rxi-17Sil MS/TG-5HT system) according to Spearman's correlation coefficient, showed lower orthogonality than systems in the classical arrangement; nevertheless, the use of separation space was considerably higher, thanks to which the maximum theoretical and conditional peak capacities were higher than in the case of classical systems. The highest conditional peak capacity was then achieved with the Rtx-200 MS/TG-5HT system. The average 2D resolution was lower than in the case of classical systems; however, the number of coelutions (7 in total) was the lowest in this case, mainly due to the much higher resolution in the second dimension than was the case in previous systems. This was also shown in the case of analyses of real samples when, in the case of Rxi-17 Sil MS/TG-5HT columns, the separation of the above-mentioned aromatic heterocyclic compounds was insufficient. In the case of the Rtx 200 MS/TG-5HT system, even these heterocyclic compounds were sufficiently separated.

The reverse system (Rtx-200 MS/TG-5HT) was evaluated as the optimal system for human scent analysis and an alternative of a $15 \mathrm{~m}$ primary column instead of a $30 \mathrm{~m}$ column was tested on this arrangement to shorten the analysis time. However, when using the $15 \mathrm{~m}$ column, the theoretical and conditional peak capacities decreased considerably, especially in the first dimension, which is due to the deterioration of the peak shape and, above all, peaks expansion.

The deterioration of the peak shape is mainly related to the long modulation periods $(15,18$, and $20 \mathrm{~s})$, which were needed to avoid the wrap-around effect. The high retention in the second dimension can be explained by the fact that, at the same temperature program, the analytes are eluted from the shorter primary column at a lower temperature, i.e., the separation on the secondary column also occurs at a lower temperature, which is associated with prolongation of retention. With a higher setting of the temperature offset between the primary and secondary ovens, there was a significant loss of resolution in the second dimension. Furthermore, it must be said that long modulation periods also correlate with long periods of cold and warm pulses (see Table 1 in Section 2.4). The problem is that short columns generate relatively narrow primary peaks, which require rapid and sharp reinjections into the secondary system to maintain properly performed modulation and cryofocusing, which was not followed in this case. The solution could be a further optimization of the modulation process, a change of the length of the secondary column, or 
change of the diameter and thickness of the stationary phase on the primary and secondary columns, respectively.

None of the systems was able to distinguish the structural isomers of higher fatty acid esters, which were represented in the model mixture by hexadecyl ester of tetradecanoic acid and dodecyl ester of octadecanoic acid (both compounds have the general formula $\mathrm{C}_{30} \mathrm{H}_{60} \mathrm{O}_{2}$ and $\mathrm{M}_{\mathrm{r}}=452.80$ ). Due to their structure and the long aliphatic chains, it would probably be necessary to use a very non-polar column, such as Rxi-1ms, to distinguish between them. In addition to changing the stationary phase, it would also probably be necessary to increase the separation efficiency, either by increasing the length of the column, which would, however, lead to very long temperature programs, or by reducing the internal diameter of the column and the thickness of the stationary phase, which in turn could cause insufficient column capacity. However, although the isomers could not be resolved chromatographically, they could be distinguished based on the characteristic masses in the mass spectrum. These esters have a typical $m / z$, which corresponds to the loss of the alkyl chain of the alcohol associated with the migration of two hydrogens.

\section{Conclusions}

Using a model mixture containing 98 compounds commonly found in human skin scent, five different column configurations were tested for GC $\times$ GC analysis. The best results were obtained for the reverse system, consisting of the $2 \mathrm{~m}$ pre-column Rtx-200 MS, the $30 \mathrm{~m}$ primary column Rtx-200 MS and the $1 \mathrm{~m}$ secondary column TG-5HT. On this reverse system, using the chromatographic method with a temperature gradient of $5{ }^{\circ} \mathrm{C} / \mathrm{min}$, the highest utilization of the separation space and the highest theoretical and conditional peak capacities of the system were achieved, and the smallest number of coelutions occurred. Therefore, this system layout will continue to be used in analyses with real scent samples.

Supplementary Materials: The following are available online at https:/ /www.mdpi.com/article/10 $.3390 /$ separations8120232/s1, Table S1: The list of the standards in a model mixture of human skin scent and measured retention indices on systems with a $2 \mathrm{~m}$ pre-column, a $30 \mathrm{~m}$ primary column, and a $1 \mathrm{~m}$ secondary column.

Author Contributions: Conceptualization, P.P. and N.L.; methodology, P.P. and N.L.; formal analysis, P.P.; resources, Š.U.; data curation, P.P.; writing—original draft preparation, P.P.; writing—review and editing, N.L. and Š.U.; supervision, Š.U.; project administration, Š.U.; funding acquisition, Š.U. All authors have read and agreed to the published version of the manuscript.

Funding: This research was funded by the Ministry of the Interior of the Czech Republic projects No.VI20172020075, VH20182021030, and VJ01010123.

Institutional Review Board Statement: This study was approved by the Institutional Review Board of University of Chemistry and Technology, Prague (approval number EK/8/2020) and complies with the Declaration of Helsinki for Medical Research involving Human Subjects.

Informed Consent Statement: The recruited volunteers were verbally instructed regarding the sampling method and analysis. They signed an agreement (written consent) regarding using his/her scent samples in this experiment and publishing the results obtained. The samples of the volunteers were used for this experiment only, and then these samples were destroyed.

Data Availability Statement: The data presented in this study are available on request from the corresponding author.

Conflicts of Interest: The authors declare no conflict of interest. The funders had no role in the design of the study; in the collection, analyses, or interpretation of data; in the writing of the manuscript; or in the decision to publish the results.

\section{Appendix A}

Cleaning of the sampling material: Sodium-calcium glass beads with a diameter of $3.6 \mathrm{~mm}$ (Glass Sphere, $\mathrm{CZ}$ ) were used for the scent collection. The glass beads were 
cleaned according to the methodology described here [12]. First, a chromium-sulfur mixture was prepared by gradually mixing $30 \mathrm{~g}$ of potassium dichromate in $0.5 \mathrm{~L}$ of concentrated sulfuric acid, in which the glass beads were placed for at least $24 \mathrm{~h}$. The excess chromium-sulfur mixture was poured through a watch glass back into the storage bottle. The remainder of the chromium-sulfur mixture was then reduced with dilute hydrogen peroxide (approximately $5 \%$ solution). This was followed by three washes with super-pure water and solvents in the order of ethanol, hexane, and again ethanol. Then, the purified glass beads were placed in an oven (Memmert UF 30 Plus, Schwabach, Germany) and dried at $200{ }^{\circ} \mathrm{C}$ for three hours. The cleaned glass beads were stored in a desiccator until use, but for a maximum time of one week.

Human skin scent sampling: Before the scent sampling, volunteers were asked to refrain from using any cosmetic products (perfumes, antiperspirants, creams, makeup, etc.) $24 \mathrm{~h}$ prior to the sampling. The scent was sampled of the volunteers' palms in the manner described herein [12]. First, the volunteers thoroughly lathered their hands and forearms with unscented Amadeus Neutral soap (Cormen, Bystřice nad Perštejnem, CZ), which they then rinsed thoroughly under warm running water. After drying their hands in the open air, the volunteers rubbed their palms together for $5 \mathrm{~min}$. Then, cleaned glass beads (70 pcs, which corresponds to about $4 \mathrm{~mL}$ ) were poured into their palms. The actual collection lasted $10 \mathrm{~min}$ and took place in such a way that the volunteers held and rubbed the cleaned glass beads in their hands. The sampled beads were then carefully transferred to a $10 \mathrm{~mL}$ headspace vial, in which a subsequent extraction took place (see Section 2.3).

\section{References}

1. Cuzuel, V.; Cognon, G.; Rivals, I.; Sauleau, C.; Heulard, F.; Thiebaut, D.; Vial, J. Origin, Analytical Characterization, and Use of Human Odor in Forensics. J. Forensic Sci. 2017, 62, 330-350. [CrossRef] [PubMed]

2. Cuzuel, V.; Sizun, A.; Cognon, G.; Rivals, I.; Heulard, F.; Thiebaut, D.; Vial, J. Human odor and forensics. Optimization of a comprehensive two-dimensional gas chromatography method based on orthogonality: How not to choose between criteria. J. Chromatogr. A 2018, 1536, 58-66. [CrossRef]

3. Woidtke, L.; Dreßler, J.; Babian, C. Individual human scent as a forensic identifier using mantrailing. Forensic Sci. Int. 2018, 282, 111-121. [CrossRef]

4. Ferry, B.; Ensminger, J.J.; Schoon, A.; Bobrovskij, Z.; Cant, D.; Gawkowski, M.; Hormila, I.; Kos, P.; Less, F.; Rodionova, E.; et al. Scent lineups compared across eleven countries: Looking for the future of a controversial forensic technique. Forensic Sci. Int. 2019, 302, 109895. [CrossRef] [PubMed]

5. Cuzuel, V.; Leconte, R.; Cognon, G.; Thiebaut, D.; Vial, J.; Sauleau, C.; Rivals, I. Human odor and forensics: Towards Bayesian suspect identification using GCxGC-MS characterization of hand odor. J. Chromatogr. B 2018, 1092, 379-385. [CrossRef]

6. de Lacy Costello, B.; Amann, A.; Al-Kateb, H.; Flynn, C.; Filipiak, W.; Khalid, T.; Osborne, D.; Ratcliffe, N.M. A review of the volatiles from the healthy human body. J. Breath Res. 2014, 8, 014001. [CrossRef]

7. Buljubasic, F.; Buchbauer, G. The scent of human diseases: A review on specific volatile organic compounds as diagnostic biomarkers. Flavour Fragr. J. 2015, 30, 5-25. [CrossRef]

8. de la Mata, A.P.; McQueen, R.H.; Nam, S.L.; Harynuk, J.J. Comprehensive two-dimensional gas chromatographic profiling and chemometric interpretation of the volatile profiles of sweat in knit fabrics. Anal. Bioanal. Chem. 2017, 409, 1905-1913. [CrossRef] [PubMed]

9. Pandey, S.K.; Kim, K.-H. Human body-odor components and their determination. TrAC Trends Anal. Chem. 2011, 30, 784-796. [CrossRef]

10. Prada, P.A.; Curran, A.M.; Furton, K.G. Comparison of extraction methods for the removal of volatile organic compounds (VOCs) present in sorbents used for human scent evidence collection. Anal. Methods 2010, 2, 470-478. [CrossRef]

11. Doležal, P.; Kyjaková, P.; Valterová, I.; Urban, Š. Qualitative analyses of less-volatile organic molecules from female skin scents by comprehensive two dimensional gas chromatography-time of flight mass spectrometry. J. Chromatogr. A 2017, 1505, 77-86. [CrossRef] [PubMed]

12. Pojmanová, P.; Ladislavová, N.; Škeříková, V.; Kania, P.; Urban, Š. Human scent samples for chemical analysis. Chem. Pap. 2020, 74, 1383-1393. [CrossRef]

13. Doležal, P.; Furton, K.G.; Lněničková, J.; Kyjaková, P.; Škeříková, V.; Valterová, I.; Pinc, L.; Urban, Š. Multiplicity of human scent signature. Egypt. J. Forensic Sci. 2019, 9, 7. [CrossRef]

14. Jha, S.K.; Hayashi, K. Molecular structural discrimination of chemical compounds in body odor using their GC-MS chromatogram and clustering methods. Int. J. Mass Spectrom. 2017, 423, 1-14. [CrossRef]

15. Colon-Crespo, L.J.; Herrera-Hernandez, D.; Holness, H.; Furton, K.G. Determination of VOC marker combinations for the classification of individuals by gender and race/ethnicity. Forensic Sci. Int. 2017, 270, 193-199. [CrossRef] [PubMed] 
16. Bakain, R.; Rivals, I.; Sassiat, P.; Thiébaut, D.; Hennion, M.-C.; Euvrard, G.; Vial, J. Comparison of different statistical approaches to evaluate the orthogonality of chromatographic separations: Application to reverse phase systems. J. Chromatogr. A 2011, 1218, 2963-2975. [CrossRef]

17. Gilar, M.; Olivova, P.; Daly, A.E.; Gebler, J.C. Orthogonality of separation in two-dimensional liquid chromatography. Anal. Chem. 2005, 77, 6426-6434. [CrossRef]

18. Davis, J.M. Dependence of Effective Peak Capacity in Comprehensive Two-Dimensional Separations on the Distribution of Peak Capacity between the Two Dimensions. Anal. Chem. 2008, 80, 8122-8134. [CrossRef] [PubMed]

19. Stoll, D.R.; Wang, X.; Carr, P.W. Comparison of the practical resolving power of one- and two-dimensional high-performance liquid chromatography analysis of metabolomic samples. Anal. Chem. 2008, 80, 268-278. [CrossRef]

20. Wang, X.; Stoll, D.R.; Schellinger, A.P.; Carr, P.W. Peak Capacity Optimization of Peptide Separations in Reversed-Phase Gradient Elution Chromatography: Fixed Column Format. Anal. Chem. 2006, 78, 3406-3416. [CrossRef]

21. Sanz, J. Chapter 3 Theoretical Considerations. In Comprehensive Analytical Chemistry; Ramos, L., Ed.; Elsevier: Amsterdam, The Netherlands, 2009; Volume 55, pp. 49-76.

22. van Den Dool, H.; Kratz, P.D. A generalization of the retention index system including linear temperature programmed gas-liquid partition chromatography. J. Chromatogr. A 1963, 11, 463-471. [CrossRef] 